\title{
Morphology-dependent electrochemical performances of nickel hydroxide nanostructures
}

\author{
KARTHIK S BHAT* ${ }^{*}$ and H S NAGARAJA \\ Department of Physics, National Institute of Technology Karnataka, P.O. Srinivasnagar, Surathkal, Mangaluru 575025 , \\ India \\ *Author for correspondence (kasubhat@gmail.com)
}

MS received 15 October 2018; accepted 8 March 2019

\begin{abstract}
Electrochemical capacitors form part of the developing technologies in the field of alternative energy sources. In the present work, nickel hydroxide $\left(\mathrm{Ni}(\mathrm{OH})_{2}\right)$ nanosheets and microflowers are hydrothermally prepared employing different chemical precursors. Structure, morphology and chemical analysis are conducted using powder X-ray diffraction, field emission scanning electron microscopy and energy-dispersive X-ray spectroscopy measurements. Electrochemical performances as supercapacitor electrodes of the synthesized nanostructures are evaluated through cyclic voltammetry and galvanostatic charge-discharge measurements with three-electrode configurations. The results indicated the specific capacitance of 180 and $417 \mathrm{~F} \mathrm{~g}^{-1}$ at a scan rate of $5 \mathrm{mV} \mathrm{s}^{-1}$ for $\mathrm{Ni}(\mathrm{OH})_{2}$ nanosheets and microflowers, respectively. The higher specific capacitances for $\mathrm{Ni}(\mathrm{OH})_{2}$ microflowers could be attributed to the higher specific surface area, morphology, electronic conductivity and porosity. Both $\mathrm{Ni}(\mathrm{OH})_{2}$ nanostructures exhibited good capacitance retention for 1500 cycles.
\end{abstract}

Keywords. Hydroxides; nickel hydroxide; supercapacitors; nanosheets; microflowers.

\section{Introduction}

The rapid surge in the energy demand and insufficiency of fossil fuels have fascinated researchers for the improvement of viable energy devices [1]. Among which supercapacitors have gained prominence owing to their ease of fabrication, composition flexibility and other analytical factors such as high power density and energy density [2,3]. Supercapacitors store charges via two mechanisms: electrochemical doublelayer capacitance (non-Faradaic) and pseudo-capacitance (Faradaic) processes [4,5]. The non-Faradic process can be ascribed to adsorption of ions at the electrode surface, while pseudo-capacitance is based on oxidation-reduction of the electrode material with the electrolyte. Graphene and their analogues exhibit electrochemical double-layer capacitance and on the other hand; materials such as metal oxides and metal hydroxides operate via redox reactions [6,7].

Various classes of nanostructures such as transition metal oxides [8], hydroxides [9], chalcogenides [10], carbon compounds [11] and conducting polymers [12] are used as electrode materials for supercapacitors. Among which transition metal hydroxides are emerged as promising electrode materials owing to their high specific surface area, redox structures and multivalent oxidation states [13]. So far, hydroxides of various transition metals such as cobalt hydroxide [14], copper hydroxide [15], lanthanum hydroxide [16], nickel hydroxide [17], manganese hydroxide [18], etc. are evaluated as electrode materials for supercapacitors. Among which, nickel hydroxide nanostructures and their carbon composites [19-24] are fairly explored with their electrochemical properties. However, no effort has been made to study the effect of the nickel hydroxide morphology on their electrochemical performances. In this framework, we account for the synthesis of nickel hydroxide nanostructures of two different morphologies (nanosheets and microflowers) via a hydrothermal method. Both nickel hydroxide nanostructures were evaluated as electrodes for supercapacitor applications. The results indicate the microflower morphology could deliver the best specific capacitance of $417 \mathrm{~F} \mathrm{~g}^{-1}$ at $5 \mathrm{mV} \mathrm{s}^{-1}$ as compared with nanosheets $\left(180 \mathrm{Fg}^{-1}\right)$ and also suggests nickel hydroxide nanostructures can be a suitable material for high-performance supercapacitor applications.

\section{Experimental}

\subsection{Chemicals and reagents}

Nickel sulphate $\left(\mathrm{NiSO}_{4} \cdot 6 \mathrm{H}_{2} \mathrm{O}\right)$, nickel nitrate $\left(\mathrm{Ni}\left(\mathrm{NO}_{3}\right)_{2}\right.$. $\left.6 \mathrm{H}_{2} \mathrm{O}\right)$, ammonium solution $\left(\mathrm{NH}_{4} \mathrm{OH}\right)$, urea $\left(\mathrm{CH}_{4} \mathrm{~N}_{2} \mathrm{O}\right)$ and trisodium citrate $\left(\mathrm{Na}_{3} \mathrm{C}_{6} \mathrm{H}_{5} \mathrm{O}_{7}\right)$ of the analytical grade available were purchased from Loba Chemicals and were used as received. Deionized (DI) water from a Milli-Q (18 M $\Omega \mathrm{cm})$ ultrapure system was used throughout the experiments. 

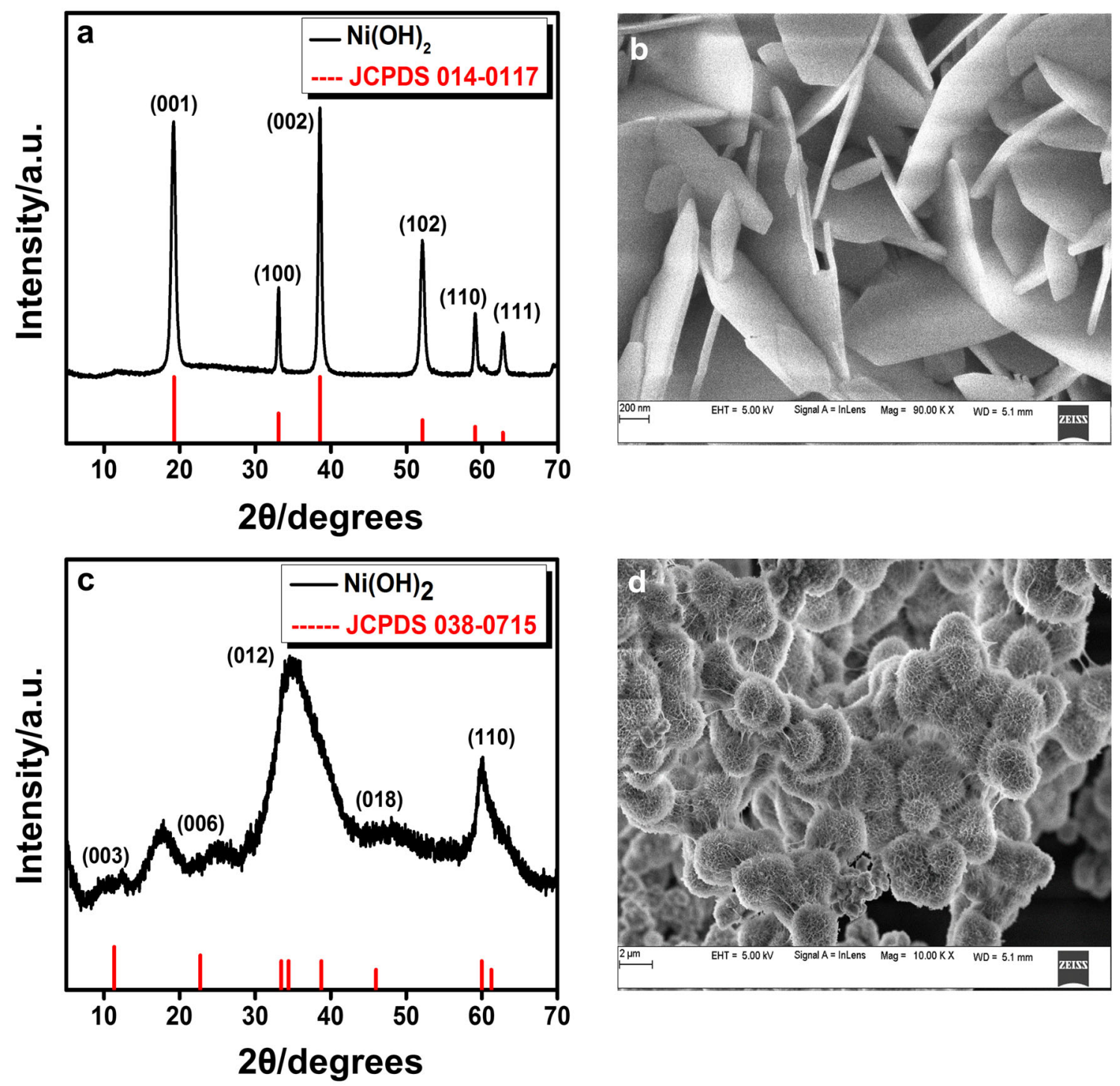

Figure 1. Powder-XRD patterns and FESEM images of: $(\mathbf{a}, \mathbf{b}) \mathrm{Ni}(\mathrm{OH})_{2}$ nanosheets, $(\mathbf{c}, \mathbf{d}) \mathrm{Ni}(\mathrm{OH})_{2}$ microflowers. (a, b) Reprinted from Bhat and Nagaraja [17], with the permission of AIP Publishing.

\subsection{Synthesis of $\mathrm{Ni}(\mathrm{OH})_{2}$ nanosheets}

The $\mathrm{Ni}(\mathrm{OH})_{2}$ nanosheets are synthesized as described elsewhere [25,26]. In brief, $0.5416 \mathrm{~g}$ of $\mathrm{NiSO}_{4} \cdot 6 \mathrm{H}_{2} \mathrm{O}$ was dissolved in $70 \mathrm{ml}$ of DI water to obtain a green solution under magnetic stirring. An opaque solution was obtained while adding $10.5 \mathrm{ml}$ of $2 \mathrm{M} \mathrm{NH}_{4} \mathrm{OH}$. The mixture was continuously stirred for $20 \mathrm{~min}$ and then transferred to a $120 \mathrm{ml}$ autoclave; the hydrothermal process was carried out at $180^{\circ} \mathrm{C}$ for $24 \mathrm{~h}$. Next, the green precipitates were collected, washed and dried at $60^{\circ} \mathrm{C}$.

\subsection{Synthesis of $\mathrm{Ni}(\mathrm{OH})_{2}$ microflowers}

The $\mathrm{Ni}(\mathrm{OH})_{2}$ microflowers are synthesized as per earlier reports [27]. In brief, $1.744 \mathrm{~g}$ of $\mathrm{Ni}\left(\mathrm{NO}_{3}\right)_{2} \cdot 6 \mathrm{H}_{2} \mathrm{O}$ and
$0.728 \mathrm{~g}$ of $\mathrm{CH}_{4} \mathrm{~N}_{2} \mathrm{O}$ were dissolved in $80 \mathrm{ml}$ of DI water to obtain an pale green solution under vigorous stirring. Then $0.024 \mathrm{~g}$ of $\mathrm{Na}_{3} \mathrm{C}_{6} \mathrm{H}_{5} \mathrm{O}_{7}$ were added to the resulting dispersion and stirred for $30 \mathrm{~min}$. The final solution was transferred to a $120 \mathrm{ml}$ autoclave and the hydrothermal process was carried out at $150^{\circ} \mathrm{C}$ for $24 \mathrm{~h}$. Dark green precipitates were collected, washed and dried at $60^{\circ} \mathrm{C}$.

\subsection{Electrode preparation and supercapacitor characterization}

The working electrodes for supercapacitors were fabricated by mixing the active material with conductive carbon black (acetylene black) and polyvinylidene fluoride in the 6:3:1 ratio, using $N$-methyl-2-pyrrolidone as the solvent. The above mixture is uniformly coated on a titanium foil substrate 

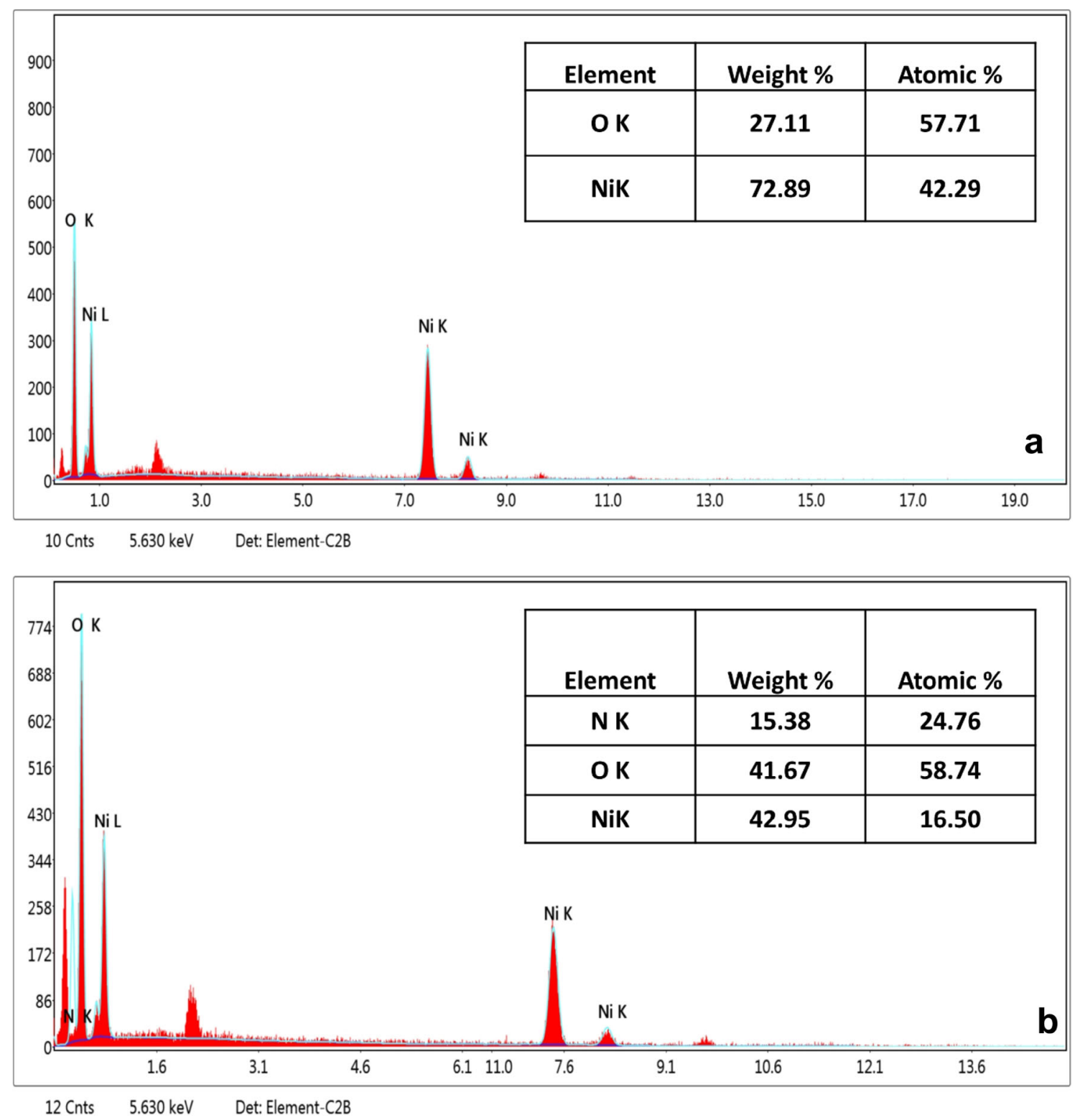

Figure 2. EDS spectra of: (a) $\mathrm{Ni}(\mathrm{OH})_{2}$ nanosheets and (b) $\mathrm{Ni}(\mathrm{OH})_{2}$ microflowers.

on $\sim 1 \mathrm{~cm}^{2}$ area and dried in an oven at $60^{\circ} \mathrm{C}$ for $6 \mathrm{~h}$. Electrochemical performances of the fabricated electrodes were evaluated using Biologic SP-150 workstation with three-electrode configurations with the fabricated electrodes as working electrodes, saturated calomel electrode (SCE) as the reference and platinum $(\mathrm{Pt})$ wire as the counter electrode. Electrochemical measurements with $1 \mathrm{M} \mathrm{KOH}$ as the electrolyte were conducted. The values of specific capacitance from the $\mathrm{CV}$ curves can be calculated using the equation:

$$
C_{\mathrm{s}}=\frac{\int I(V) \mathrm{d} V}{v m \Delta V}\left(\mathrm{Fg}^{-1}\right),
$$

where ' $C_{\mathrm{s}}$ ' represents the specific capacitance $\left(\mathrm{F} \mathrm{g}^{-1}\right)$, integral represents the area under the CV curve, ' $m$ ' is the active mass of the electroactive material (mg), ' $v$ ' is the scan rate $\left(\mathrm{mV} \mathrm{s}^{-1}\right)$ and ' $\Delta V$ ' is the potential window $(\mathrm{V})$.

\subsection{Structure, morphology and chemical analysis}

Figure 1a and $\mathrm{b}$ shows powder-X-ray diffraction (PXRD) patterns and field emission scanning electron microscopy (FESEM) images of $\mathrm{Ni}(\mathrm{OH})_{2}$ nanosheets and figure $1 \mathrm{c}$ and d shows PXRD patterns and FESEM images of $\mathrm{Ni}(\mathrm{OH})_{2}$ microflowers.

Figure 1a shows the XRD patterns of the as-synthesized $\mathrm{Ni}(\mathrm{OH})_{2}$ nanosheets with lattice constants $a=2.12 \AA$ and 

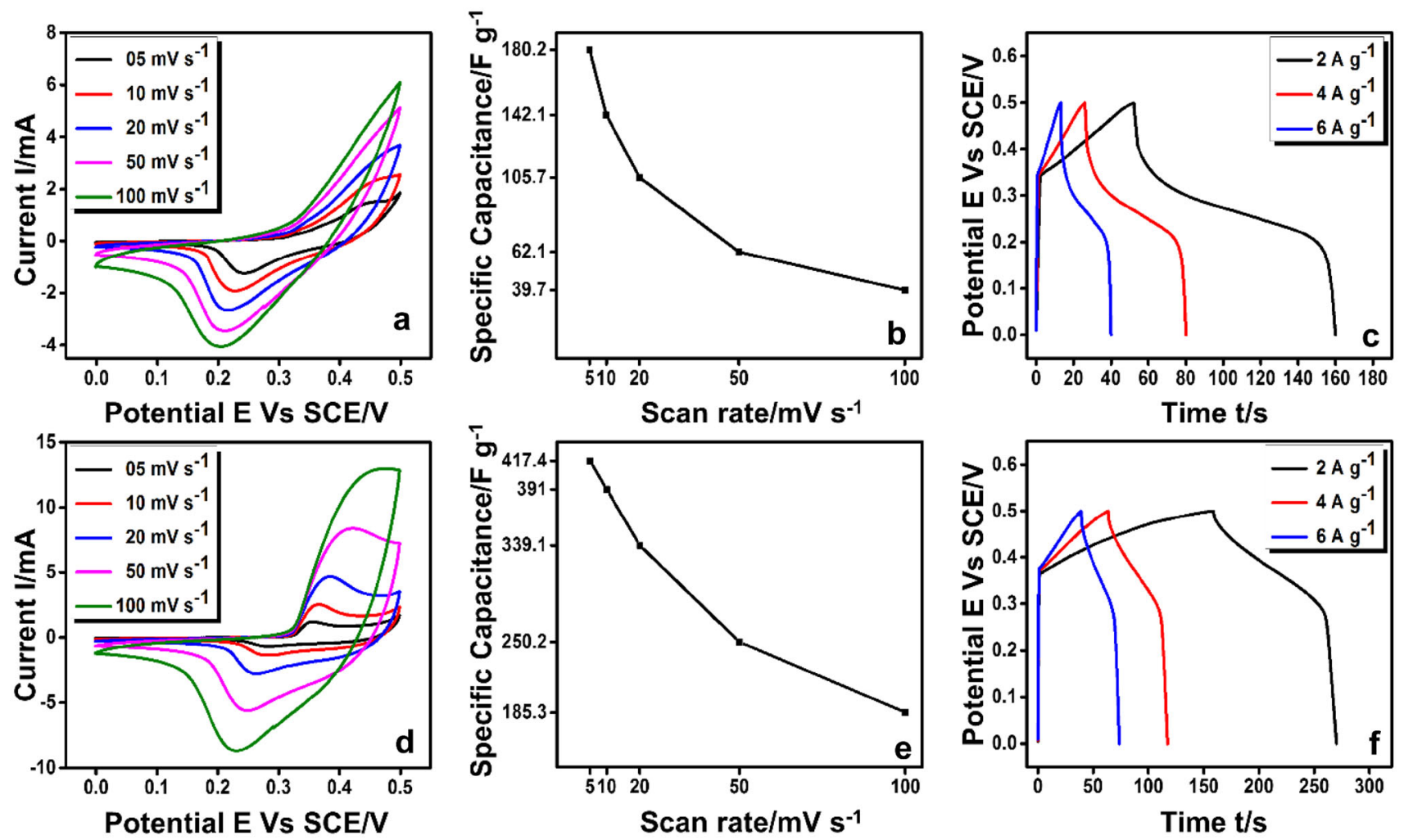

Figure 3. (a, d) $\mathrm{CV}$ curves of $\mathrm{Ni}(\mathrm{OH})_{2}$ nanosheets and microflowers at various scan rates; (b, e) effect of the scan rate on the specific capacitances of $\mathrm{Ni}(\mathrm{OH})_{2}$ nanosheets and microflowers and $(\mathbf{c}, \mathbf{f}) \mathrm{GCD}$ curves of $\mathrm{Ni}(\mathrm{OH})_{2}$ nanosheets and microflowers at different current densities. (a-c) Reprinted from Bhat and Nagaraja [17], with the permission of AIP Publishing.

Table 1. Specific capacitance values at different scan rates of $\mathrm{Ni}(\mathrm{OH})_{2}$ nanosheets and microflowers.

\begin{tabular}{rcc}
\hline & \multicolumn{2}{c}{ Specific capacitance $\left(\mathrm{F} \mathrm{g}^{-1}\right)$} \\
\cline { 2 - 3 } Scan rate $\left(\mathrm{mV} \mathrm{s}^{-1}\right)$ & Nanosheets & Microflowers \\
\hline 5 & 180.2 & 417.4 \\
10 & 142.1 & 391.0 \\
20 & 105.6 & 339.1 \\
50 & 62.1 & 250.2 \\
100 & 39.7 & 185.3 \\
\hline
\end{tabular}

$c=4.60 \AA$ with a hexagonal crystal structure, which are commensurate with standard JCPDS files 014-0117 [28]. On the other hand, figure 1c represents the XRD patterns of $\mathrm{Ni}(\mathrm{OH})_{2}$ microflowers indicating the rhombohedral crystal structure with lattice constants $a=b=3.08 \AA$ and $c=23.4 \AA$, which are consistent with reference JCPDS file 038-0715 [29]. Further, FESEM measurements confirm the two-dimensional hexagonal sheet-like morphology of $\mathrm{Ni}(\mathrm{OH})_{2}$ nanosheets (figure 1b) and the interconnected threedimensional flower-like network of $\mathrm{Ni}(\mathrm{OH})_{2}$ microflowers (figure 1d), respectively.

Next, energy-dispersive X-ray spectroscopy (EDS) measurements were performed to determine the elemental composition of the structures as shown in figure $2 \mathrm{a}$ and $\mathrm{b}$. Both $\mathrm{Ni}(\mathrm{OH})_{2}$ nanosheet and microflower morphologies indicate the elemental peaks corresponding to $\mathrm{Ni}$ and $\mathrm{O}$, confirming their purity. Surprisingly, the EDS measurements on $\mathrm{Ni}(\mathrm{OH})_{2}$ microflowers also indicate the peaks corresponding to nitrogen, which could be due to the use of a urea precursor during the synthesis process. Since the presence of nitrogen is in considerable quantities, one can assume the nitrogen doping in the $\mathrm{Ni}(\mathrm{OH})_{2}$ microflower system. Further, the atomic and weight percentages obtained from EDS measurements are provided as insets of figure $2 a$ and $b$, respectively.

\subsection{Electrochemical measurements}

The electrochemical performances of fabricated $\mathrm{Ni}(\mathrm{OH})_{2}$ supercapacitor electrodes are evaluated using cyclic voltammetry (CV), galvanostatic charge-discharge (GCD) and electrochemical impedance spectroscopy (EIS) techniques. All electrochemical measurements are performed in threeelectrode configurations with $1 \mathrm{M} \mathrm{KOH}$ as the aqueous electrolyte. CV and GCD curves were recorded in the voltage window range of $0-0.5 \mathrm{~V} v s$. SCE at various scan rates $\left(5-100 \mathrm{mV} \mathrm{s}^{-1}\right)$ and different applied current densities $\left(2,4\right.$ and $\left.6 \mathrm{~A} \mathrm{~g}^{-1}\right)$, respectively. Further, EIS measurements were performed in the frequency range of $0.2 \mathrm{~Hz}-100 \mathrm{kHz}$ to 

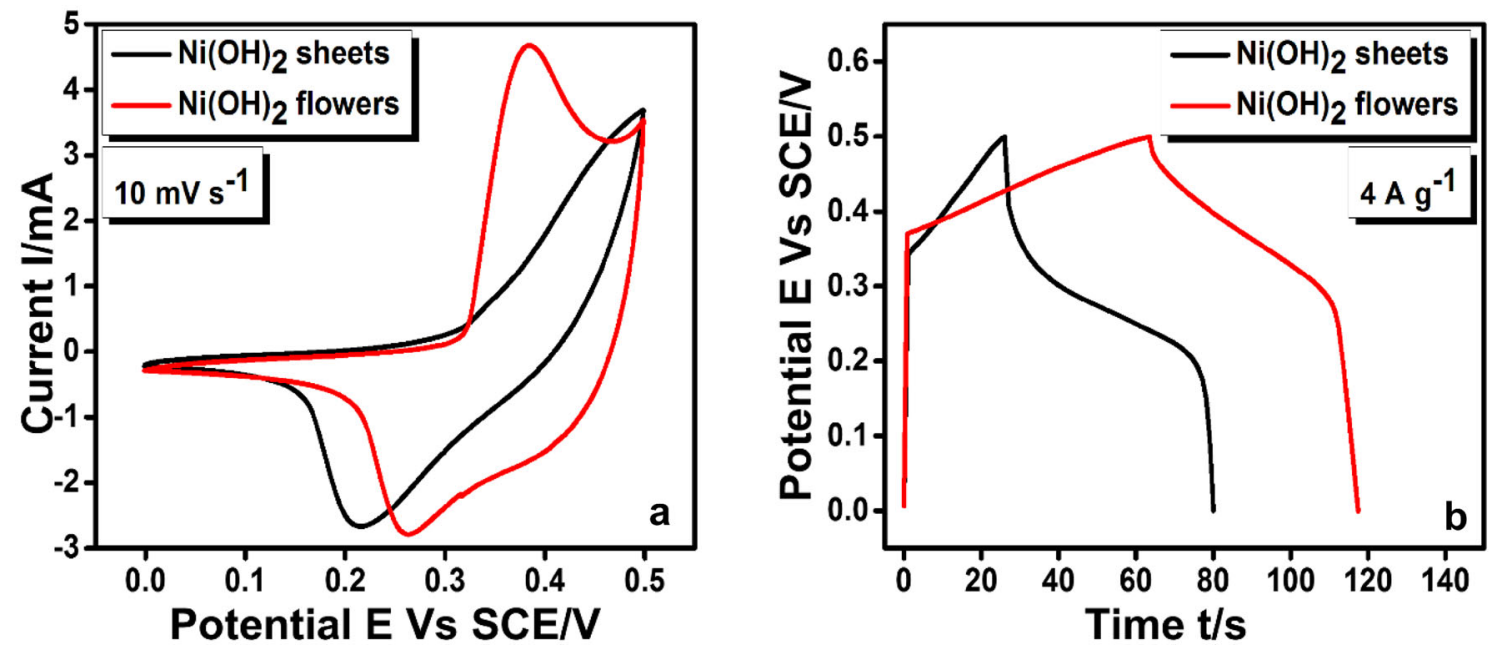

Figure 4. Comparison of (a) $\mathrm{CV}$ curves and (b) $\mathrm{GCD}$ curves of $\mathrm{Ni}(\mathrm{OH})_{2}$ nanosheet and microflower electrodes at a scan rate of $10 \mathrm{mV} \mathrm{s}^{-1}$ and a current density of $4 \mathrm{~A} \mathrm{~g}^{-1}$ respectively.

study the interface behaviour of the electrode material with the electrolyte.

Figure $3 \mathrm{a}$ and $\mathrm{d}$ shows $\mathrm{CV}$ curves of $\mathrm{Ni}(\mathrm{OH})_{2}$ nanosheets and microflowers at various scan rates, figure $3 b$ and e shows the effect of a scan rate on the specific capacitances of $\mathrm{Ni}(\mathrm{OH})_{2}$ nanosheets and microflowers and figure $3 \mathrm{c}$ and $\mathrm{f}$ shows $\mathrm{GCD}$ curves of $\mathrm{Ni}(\mathrm{OH})_{2}$ nanosheets and microflowers at different current densities.

Figure $3(\mathrm{a}$ and $\mathrm{d})$ represents the $\mathrm{CV}$ curves of the fabricated $\mathrm{Ni}(\mathrm{OH})_{2}$ nanosheets and microflowers in the voltage window range of $0-0.5 \mathrm{~V}$. The $\mathrm{CV}$ curves differ from the ideal rectangular behaviour of the supercapacitor, suggesting the redox-type charge storage behaviour of the electrodes. In other words, both nanostructures exhibit pseudo-capacitive characteristics [30]. Also, it is important to consider that the $\mathrm{Ni}(\mathrm{OH})_{2}$ microflowers exhibit prominent oxidation and reduction peaks in contrast to $\mathrm{Ni}(\mathrm{OH})_{2}$ nanosheets, suggesting the good reversibility and higher specific capacitances of the electrodes. Further, the values of specific capacitances at different scan rates as estimated from $\mathrm{CV}$ curves using equation (1) are tabulated in table 1.

The steady decrease in the specific capacitance at a higher scan rate can be attributed to the inadequate time for adsorption and diffusion of electrolyte ions at the electrode surface. The variation in specific capacitances of $\mathrm{Ni}(\mathrm{OH})_{2}$ nanosheets and microflowers with respect to scan rates is shown in figure $3 \mathrm{~b}$ and e, respectively. It is worth noting that even at higher scan rates $\left(100 \mathrm{mV} \mathrm{s}^{-1}\right) \mathrm{Ni}(\mathrm{OH})_{2}$ microflowers retain about $\sim 44 \%$ of the initial capacitance $\left(417 \mathrm{~F} \mathrm{~g}^{-1}\right.$ at a scan rate of $5 \mathrm{mV} \mathrm{s}^{-1}$ ) suggesting the prominent electrochemical stability of the electrodes.

The value of the specific capacitance of $\mathrm{Ni}(\mathrm{OH})_{2}$ microflower electrodes is comparatively higher as compared with $\mathrm{Ni}(\mathrm{OH})_{2}$ nanosheet electrodes. For instance at a scan rate of $5 \mathrm{mV} \mathrm{s}^{-1}$, the obtained value of the specific capacitance

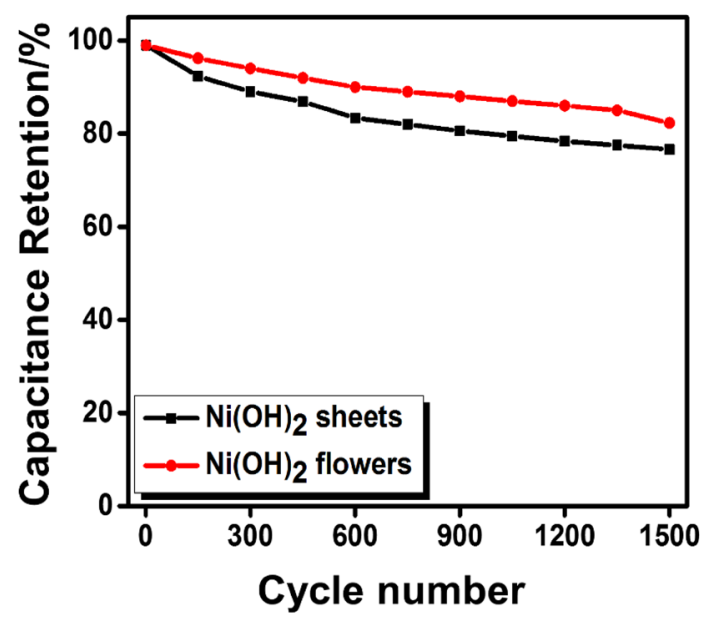

Figure 5. Cyclic stability of $\mathrm{Ni}(\mathrm{OH})_{2}$ nanosheet and microflower electrodes.

for $\mathrm{Ni}(\mathrm{OH})_{2}$ microflowers is $417 \mathrm{~F} \mathrm{~g}^{-1}$, which is more than a twice as compared with $\mathrm{Ni}(\mathrm{OH})_{2}$ nanosheets $\left(180 \mathrm{~F} \mathrm{~g}^{-1}\right)$. The large difference in the specific capacitance maybe attributed to the higher specific surface area and pore size of the $\mathrm{Ni}(\mathrm{OH})_{2}$ microflowers, which in turn enhances the adsorption and diffusion of electrolyte ions during the cyclic process.

Further, figure $3 \mathrm{c}$ and $\mathrm{f}$ depicts the $\mathrm{GCD}$ curves of $\mathrm{Ni}(\mathrm{OH})_{2}$ nanosheets and microflowers. The deviation from the typical triangular charge-discharge profile confirms the pseudocapacitive nature of the electrodes. It can also be noted that the charge-discharge time of $\mathrm{Ni}(\mathrm{OH})_{2}$ microflowers is higher as compared with $\mathrm{Ni}(\mathrm{OH})_{2}$ nanosheets, which once again confirms the higher values of specific capacitances of the $\mathrm{Ni}(\mathrm{OH})_{2}$ microflower electrodes as estimated from CV curves. For better comparison of the electrochemical performances, $\mathrm{CV}$ 

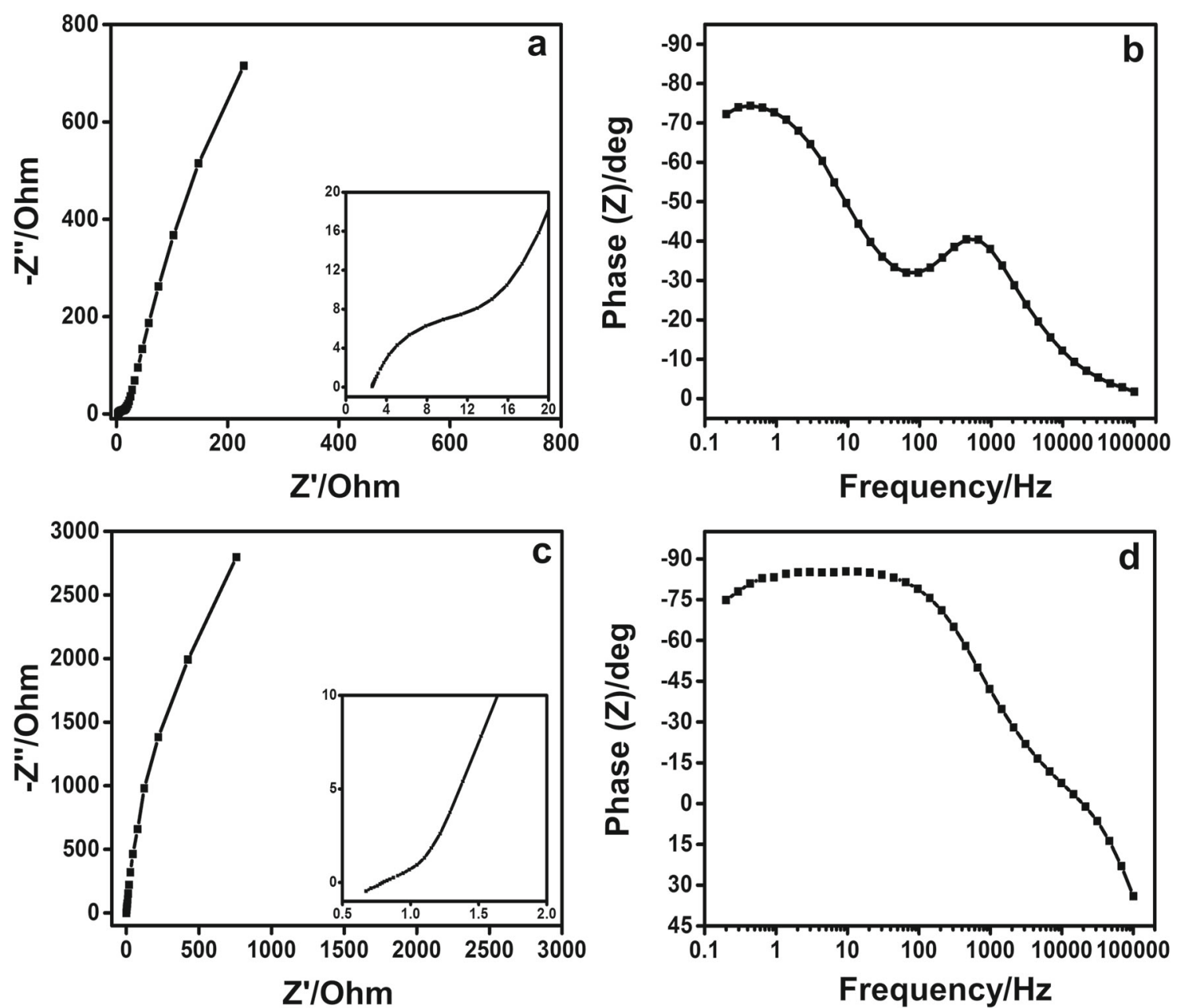

Figure 6. (a, c) Nyquist plots of $\mathrm{Ni}(\mathrm{OH})_{2}$ nanosheets and microflowers and their corresponding Bode phase angle plots (b, d). (a, b) Reprinted from Bhat and Nagaraja [17], with the permission of AIP Publishing.

Table 2. Parameters obtained from the Nyquist plots of the electrodes.

\begin{tabular}{lcc}
\hline EIS values & Nanosheets & Microflowers \\
\hline$R_{\mathrm{S}}(\Omega)$ & 2.58 & 0.68 \\
$R_{\mathrm{CT}}(\Omega)$ & 10.42 & 0.42 \\
\hline
\end{tabular}

curves at a scan rate of $10 \mathrm{mV} \mathrm{s}^{-1}$ and GCD curves at a current density of $4 \mathrm{~A} \mathrm{~g}^{-1}$ are shown in figure $4 \mathrm{a}$ and $\mathrm{b}$, respectively.

The cycle lifespan is one of the important parameters of supercapacitor applications. We have examined the electrochemical stability of the fabricated both $\mathrm{Ni}(\mathrm{OH})_{2}$ nanosheets and microflower-based electrodes through $\mathrm{CV}$ measurements at a scan rate of $50 \mathrm{mV} \mathrm{s}^{-1}$ for 1500 cycles. As shown in figure 5 , the results indicate the specific capacitance retention of 76 and $82 \%$ for $\mathrm{Ni}(\mathrm{OH})_{2}$ nanosheets and microflowers, respectively, indicating the promising stability of the electrodes.
Finally, the interface behaviour of the $\mathrm{Ni}(\mathrm{OH})_{2}$ supercapacitor electrodes is characterized with the help of EIS measurements. EIS measurements were performed in the frequency range of $0.2 \mathrm{~Hz}-100 \mathrm{kHz}$, with the applied bias of $10 \mathrm{mV}$. Supercapacitors behave as resistors at high frequencies, drawing a kinetic arc and capacitive behaviour at low frequencies [31], presenting a vertical straight line [32]. The vertical straight line also indicates the prominent capacitance response and good electronic conductivity of the materials [33]. Figure 6a and c represents the recorded Nyquist plots and figure $6 \mathrm{~b}$ and $\mathrm{d}$ depicts the Bode phase angle plot of $\mathrm{Ni}(\mathrm{OH})_{2}$ nanosheet and microflower electrodes.

The parameters extracted from the Nyquist plots consist of electrode resistance $\left(R_{\mathrm{S}}\right)$ and charge transfer resistance $\left(R_{\mathrm{CT}}\right)$. The lower $R_{\mathrm{S}}$ value indicates the better contact of electrodes with the electrolyte and the lower $R_{\mathrm{CT}}$ indicates the higher electronic conductivity and higher rate of adsorption/diffusion of electrolyte ions through the electrodes [34]. The value of $R_{\mathrm{CT}}$ is as low as $0.42 \Omega$ for $\mathrm{Ni}(\mathrm{OH})_{2}$ microflowers and the value of $R_{\mathrm{S}}$ is about three times 
lower as compared with $\mathrm{Ni}(\mathrm{OH})_{2}$ nanosheets, once again supporting the higher values of specific capacitances. The parameters extracted from the Nyquist plots are tabulated in table 2.

Further, the Bode phase angle is also the important parameter of the supercapacitors. Ideal capacitors display the phase angle of $-90^{\circ}$, while the phase angle varies for pseudocapacitors [35]. The obtained phase angles are -75 and $-85^{\circ}$ for $\mathrm{Ni}(\mathrm{OH})_{2}$ nanosheets and microflowers, which also confirm the pseudocapacitive charge storage mechanism of the electrodes.

\section{Conclusion}

In summary, $\mathrm{Ni}(\mathrm{OH})_{2}$ nanostructures of two different morphologies (nanosheets and microflowers) were synthesized employing a hydrothermal method. When used as the electrodes for supercapacitors, the $\mathrm{Ni}(\mathrm{OH})_{2}$ microflowers could deliver the enhanced specific capacitance of $417.4 \mathrm{~F} \mathrm{~g}^{-1}$ at a scan rate of $5 \mathrm{mV} \mathrm{s}^{-1}$, which is more than twice as compared to $\mathrm{Ni}(\mathrm{OH})_{2}$ nanosheets with a specific capacitance of $180 \mathrm{~F} \mathrm{~g}^{-1}$. Both morphologies of $\mathrm{Ni}(\mathrm{OH})_{2}$ exhibited good capacitance retention capability for $1500 \mathrm{CV}$ cycles. The superior electrochemical performance of the nanostructures opens up interesting possibilities for the design and development of high-performance energy storage devices.

\section{References}

[1] Serrano E, Rus G and García-Martínez J 2009 Renewable Sustainable Energy Rev. 132373

[2] Wang H, Feng H and Li J 2014 Small 102165

[3] Hu Y and Fisher T S 2018 Bull. Mater. Sci. 41124

[4] Zhi M, Xiang C, Li J, Li M and Wu N 2013 Nanoscale 572

[5] Bhat K S and Nagaraja H S 2019 Electrochim. Acta 302459

[6] Li W and Yang Y J 2014 J. Solid State Electrochem. 181621

[7] Gao Y, Wu J, Zhang W, Tan Y, Gao J, Tang B et al 2015 J. Appl. Electrochem. 45541

[8] Periasamy P, Krishnakumar T, Sathish M, Chavali M, Siril P F and Devarajan V P 2018 J. Mater. Sci.: Mater. Electron. 29 6157

[9] Sichumsaeng T, Chanlek N and Maensiri S 2018 Appl. Surf. Sci. 446177
[10] Bhat K S, Shenoy S, Nagaraja H S and Sridharan K 2017 Electrochim. Acta $\mathbf{2 4 8} 188$

[11] Chen C, Fan W, Ma T and Fu X 2014 Ionics 201489

[12] Tamai H, Hakoda M, Shiono T and Yasuda H 2007 J. Mater. Sci. 421293

[13] Kiran S K, Padmini M, Das H T and Elumalai P 2017 J. Solid State Electrochem. 21927

[14] Aghazadeh M, Karimzadeh I, Ahmadi A and Ganjali M R 2018 J. Mater. Sci.: Mater. Electron. 2914567

[15] Ghasemi S, Jafari M and Ahmadi F 2016 Electrochim. Acta 210 225

[16] Rajagopal R and Ryu K-S 2018 J. Ind. Eng. Chem. 60441

[17] Bhat K S and Nagaraja H S 2018 AIP Conf. Proc. 1943 020057

[18] Anandan S, Gnana Sundara Raj B, Lee G-J and Wu J J 2013 Mater. Res. Bull. 483357

[19] Kovalenko V L, Kotok V A, Sykchin A A, Mudryi I A, Ananchenko B A, Burkov A A et al 2017 J. Solid State Electrochem. 21683

[20] Chen Y, Zhang Z, Sui Z, Liu Z, Zhou J and Zhou X 2016 Int. J. Hydrogen Energy 4112136

[21] Qu R, Tang S, Qin X, Yuan J, Deng Y, Wu L et al 2017 J. Alloys Compd. $\mathbf{7 2 8} 222$

[22] Wang H, Shi X, Zhang W and Yao S 2017 J. Alloys Compd. 711 643

[23] Zeng Z, Sun P, Zhu J and Zhu X 2017 Surf. Interfaces 873

[24] Ghosh D, Giri S, Mandal A and Das C K 2013 Chem. Phys. Lett. 57341

[25] Bhat K S, Barshilia H C and Nagaraja H S 2017 Int. J. Hydrogen Energy 4224645

[26] Karthik S B and Nagaraja H S 2018 Mater. Res. Express 5 105504

[27] Guan B, Li Y, Yin B, Liu K, Wang D, Zhang H et al 2017 Chem. Eng. J. 3081165

[28] Bhat K S and Nagaraja H S 2018 Int. J. Hydrogen Energy 43 19851

[29] Kim S-I, Kang K-N, Kim S-W and Jang J-H 2014 RSC Adv. 4 59310

[30] Bu I Y Y and Huang R 2015 Mater. Sci. Semicond. Process. 31 131

[31] Portet C, Taberna P L, Simon P, Flahaut E and Laberty-Robert C 2005 Electrochim. Acta $\mathbf{5 0} 4174$

[32] Aurbach D 1998 J. Electrochem. Soc. 1453024

[33] Musiani M M 1990 Electrochim. Acta 351665

[34] Zhu J, Xiang L, Xi D, Zhou Y and Yang J 2018 Bull. Mater. Sci. 4154

[35] Biswal M, Banerjee A, Deo M and Ogale S 2013 Energy Environ. Sci. 61249 